\title{
Effects of coadministration of natural polyphenols with doxycycline or calcium modulators on acute Chlamydia pneumoniae infection in vitro
}

\author{
Olli P Salin ${ }^{1}$, Leena L Pohjala ${ }^{1}$, Pekka Saikku², Heikki J Vuorela ${ }^{3}$, Maija Leinonen ${ }^{4}$ and Pia M Vuorela ${ }^{1}$
}

Besides small molecules from medicinal chemistry, natural products are still major sources of innovative therapeutic agents for various conditions, including infectious diseases. Here we present the first attempt to design a combination treatment targeted against Chlamydia pneumoniae infection using coadministration of natural phenolics with calcium $\left(\mathrm{Ca}^{2+}\right)$ modulators, and also the concomitant administration of these compounds with doxycycline. An in vitro acute $C$. pneumoniae model in human lung epithelial cells was used and Loewe additivity model was applied to evaluate the effects. In general, the phenolic compounds, quercetin, luteolin, rhamnetin and octyl gallate did not improve the antichlamydial effect of doxycycline, and, in some cases, resulted in antagonistic effects. The combination of doxycycline and $\mathrm{Ca}^{2+}$ modulators (isradipine, verapamil and thapsigargin) was at most additive, and at subinhibitory concentrations of doxycycline, often even antagonistic. The $\mathrm{Ca}^{2+}$ modulators showed no inhibitory effects on $\boldsymbol{C}$. pneumoniae growth alone, whereas the coadminstration of $\mathrm{Ca}^{2+}$ modulators with phenolic compounds resulted in potentiation of the antichlamydial effect of phenolic compounds. Verapamil ( $100 \mu \mathrm{m})$ was synergistic with low quercetin and luteolin concentrations $(0.39$ and $1.56 \mu \mathrm{m})$, whereas $10 \mu \mathrm{m}$ isradipine was synergistic with high quercetin, rhamnetin and octyl gallate concentrations $(12.5 \mu \mathrm{m}$ and $100 \mu \mathrm{m})$. Use of thapsigargin with the phenolic compounds resulted in the most intense synergism. Interaction indices 0.12 and 0.14 were achieved with $0.39 \mu \mathrm{m}$ luteolin and 10 and $100 \mathrm{~nm}$ thapsigargin, respectively. To conclude, the observed results indicate that the $\mathrm{Ca}^{2+}$ modulators potentiate the antichlamydial effects of the phenolic compounds.

The Journal of Antibiotics (2011) 64, 747-752; doi:10.1038/ja.2011.79; published online 21 September 2011

Keywords: antimicrobial effect; Chlamydia; phenolic compound; synergy

\section{INTRODUCTION}

Chlamydia pneumoniae is an intracellular Gram-negative bacterium infecting readily the epithelial and smooth muscle cells, as well as monocytes and macrophages. The bacterium causes acute respiratory tract infections, and is estimated to be responsible for $5-10 \%$ of community-acquired pneumonia. ${ }^{1}$ The persistent infection of C. pneumoniae has been associated with the development of several inflammatory diseases. For example, the association between Chlamydia pneumoniae and atherosclerosis has been indicated by several approaches including in vivo animal studies, seroepidemiological studies in humans, demonstration of the presence of the bacteria in atherosclerotic lesions and atherosclerotic changes in vascular tissue. $^{2-5}$

Antimicrobial therapy is heading toward multidrug treatments because microbial resistance toward commonly used antibiotics has increased. ${ }^{6}$ The multidrug treatment approach has already been used for long against difficult pathogens that typically develop resistance to antibiotics such as Mycobacterium tuberculosis or respond poorly to single-drug treatment such as Helicobacter pylori. Moreover, in serious cases of C. pneumoniae infection, such as Chlamydia-induced arthritis, prolonged treatment with multiple antibiotics has been successfully used. $^{7}$

Most antibiotics limit infections by inhibiting central microbial biosynthetic pathways, thus having relatively little effect on the metabolically inactive forms of pathogens. ${ }^{8}$ Occasionally, in vivo antibiotic treatment of acute $C$. pneumoniae infections with standard clinical doses has failed. ${ }^{9}$ These treatment failures may promote the development of persistent infection. In fact, some of the first-choice antibiotics, such as doxycycline and rifampicin, have been reported to promote the development of persistent infection at subinhibitory concentrations. ${ }^{10}$ It has been suggested that $C$. pneumoniae infection of macrophages is often in a persistent form, which explains the observed refractory behavior of $C$. pneumoniae infection to antibiotic treatment in cells of monocyte-macrophage line. ${ }^{11,12}$ Thus, new

${ }^{1}$ Pharmaceutical Sciences, Department of Biosciences, Abo Akademi University, Turku, Finland; ${ }^{2}$ Institute of Diagnostics, Department of Medical Microbiology, University of Oulu, Oulu, Finland; ${ }^{3}$ Division of Pharmaceutical Biology, Faculty of Pharmacy, University of Helsinki, Helsinki, Finland and ${ }^{4}$ National Institute for Health and Welfare (THL), Oulu, Finland

Correspondence: Professor PM Vuorela, Pharmaceutical Sciences, Department of Biosciences, Abo Akademi University, BioCity, Artillerigatan 6 A, Turku FI-20520, Finland. E-mail: pia.vuorela@abo.fi

Received 13 May 2011; revised 26 July 2011; accepted 28 July 2011; published online 21 September 2011 
treatments are needed to fight the chlamydial infections and avoid the treatment failures.

Calcium $\left(\mathrm{Ca}^{2+}\right)$ channel blockers have been shown to inhibit the growth of some extracellular and intracellular pathogens and to have combinatory effects with certain antimicrobials. ${ }^{13}$ High doses of verapamil have been shown to inhibit the growth of Chlamydia, ${ }^{14}$ and most interestingly, $\mathrm{Ca}^{2+}$ channel blockers have been shown to improve antibiotic susceptibility of persistent $C$. pneumoniae infection, presumably by reactivating the persistent infection to more easily treatable acute infection. ${ }^{15,16}$

We have previously shown that phenolic compounds, common in our daily food, are potent and valid inhibitors of C. pneumoniae, in vitro and in vivo, even with doses achievable from the nutritional sources. ${ }^{17,18}$ Phenolic compounds have been associated with a wide range of biological activities; among others, they have been shown to affect $\mathrm{Ca}^{2+}$ channel regulation in mammalian cells at physiological concentrations. As reviewed by Scholz et al., ${ }^{19}$ the type and strength of the effect depends highly on the compound concentration and on the cell and channel type. In our earlier work with rat pituitary $\mathrm{GH}_{4} \mathrm{C}_{1}$ cells expressing L-type $\mathrm{Ca}^{2+}$ channels, luteolin and octyl gallate inhibited the uptake of $\mathrm{Ca}^{2+}$ from extracellular media, whereas quercetin increased the uptake, and rhamnetin showed no effect on the $\mathrm{Ca}^{2+}$ ion uptake at a concentration of $20 \mu \mathrm{g} \mathrm{ml}^{-1}(63-71 \mu \mathrm{M}){ }^{20}$ These phenolic compounds sharing good antichlamydial activity, but showing differential $\mathrm{Ca}^{2+}$-modulating activities, were chosen for the current study.

In this study we investigated whether coadministration of doxycycline, $\mathrm{Ca}^{2+}$ modulators and phenolic compounds in appropriate dose could produce a synergistic antimicrobial effect on acute chlamydial infection in vitro. Two clinically used $\mathrm{Ca}^{2+}$ channel blockers were used, isradipine being highly selective inhibitor of L-type $\mathrm{Ca}^{2+}$ channels and verapamil, which is relatively non-selective $\mathrm{Ca}^{2+}$ channel inhibitor. In addition, thapsigargin, a selective inhibitor of the sarco/endoplasmic reticulum $\mathrm{Ca}^{2+}$-ATPase pump was included in the study.

\section{RESULTS}

Dose-dependent inhibition of $C$. pneumoniae growth by single compounds

First, the dose-dependence of the antichlamydial effect of $\mathrm{Ca}^{2+}$ channel blockers, isradipine and verapamil, sarco/endoplasmic reticulum $\mathrm{Ca}^{2+}$-ATPase pump, inhibitor thapsigargin, doxycycline, the three flavonoids quercetin, luteolin and rhamnetin, and an alkyl gallate octyl gallate, were studied in a model for acute C. pneumoniae infection in human lung epithelial (HL) cells.

The treatment of the chlamydial cultures with $\mathrm{Ca}^{2+}$ channel blockers (up to $100 \mu \mathrm{m}$ concentration) had minor or moderate effects on the number of chlamydial inclusions. Isradipine was the more efficient from the two tested $\mathrm{Ca}^{2+}$ channel blockers in reducing chlamydial growth, as treatment with $10 \mu \mathrm{M}$ isradipine resulted in $56 \%$ inhibition of chlamydial growth, and with $100 \mu \mathrm{M}$ isradipine, $87 \%$ inhibition of number of chlamydial inclusions was achieved, yet this higher concentration also decreased host cell viability to $54 \%$. (Table 1) Verapamil, on the other hand, gave $28 \%$ inhibition of chlamydial growth at a concentration of $100 \mu \mathrm{M}$, whereas treatment with $10 \mu \mathrm{m}$ concentration markedly increased the number of chlamydial inclusions. Lower concentrations of verapamil resulted in no significant change in the number of chlamydial inclusions. Thapsigargin showed $53 \%$ inhibition at $1 \mu \mathrm{M}$ concentration, but also decreased HL cell viability at this concentration. Lower thapsigargin concentrations showed no antichlamydial effects.

The treatment of chlamydial cultures with doxycycline, quercetin, luteolin, rhamnetin and octyl gallate resulted in dose-dependent inhibition of chlamydial growth as measured by the number of chlamydial inclusions. Sigmoidal dose-response curves for the antichlamydial effects of doxycycline, the three flavonoids and octyl gallate were obtained (data not shown). The $\mathrm{IC}_{50}$ values determined by the curves were $49 \mathrm{~nm}\left(\log \mathrm{IC}_{50}-7.3 \pm 0.26\right)$ for doxycycline, $46 \mu \mathrm{M}$ $(-4.3 \pm 0.17)$ for quercetin, $16 \mu \mathrm{M}(-4.8 \pm 0.26)$ for luteolin, $50 \mu \mathrm{M}$ $(-4.3 \pm 0.25)$ for rhamnetin and $4.2 \mu \mathrm{M}(-5.4 \pm 0.18)$ for octyl gallate.

The inhibition of $C$. pneumoniae by combinations with doxycycline Doxycycline (70, 35 and $18 \mathrm{~nm}$ ) was combined with the three flavonoids and octyl gallate. When combined with quercetin, most samples within the combination treatments gave higher inclusion numbers than untreated controls, resulting in negative inhibition percentages; however, the inhibition values of both doxycycline and quercetin alone were consistent with the obtained dose-response curves. As a result of the negative inhibition values obtained for most of the combinations, the interaction indices $(I)$ calculated for the different doxycycline-quercetin combination showed mostly strongto-moderate antagonism ( $I$-values $>2$, in most combinations) (Table 2). However, an $I$-value of 0.9 was achieved with $70 \mathrm{~nm}$ doxycycline $+6.25 \mu \mathrm{M}$ quercetin combination, indicating an additive effect.

Similar data were obtained when doxycycline was combined with the two other flavonoids, luteolin and rhamnetin. Most of the samples treated with the combinations in different doses resulted in negative inhibition values and thus calculated $I$-values indicate strong antagonizing effects between the agents. Moreover, doxycycline-octyl gallate combinations showed some negative inhibition; antagonizing or indifferent rather than additive or synergistic effects for all the concentration combinations (Table 2).

Table 1 Effect of the studied compounds on HL cell viability (ATP level), measured with a commercial kit CellTiter Glo (Promega, Madison, WI, USA) after $70 \mathrm{~h}$ exposure

Viability \% (s.e.m. \%)

\begin{tabular}{|c|c|c|c|c|c|c|c|}
\hline Concentration ( $\mu \mathrm{M})$ & Quercetin & Luteolin & Rhamnetin & Octyl gallate & Thapsigargin & Verapamil & Isradipine \\
\hline 100 & $89(4.6)$ & $47(3.8)$ & $81(1.0)$ & $21(39.7)$ & - & $81(0.4)$ & $54(0.8)$ \\
\hline 10 & $109(2.7)$ & $89(1.2)$ & $89(10.2)$ & $97(1.8)$ & - & $81(0.3)$ & $81(1.0)$ \\
\hline 1 & 105 (1.0) & $102(1.0)$ & $99(1.0)$ & $109(0.5)$ & $61(0.8)$ & $82(0.2)$ & $79(1.5)$ \\
\hline 0.1 & $101(0.5)$ & $102(1.2)$ & $101(1.0)$ & $111(0.6)$ & $68(3.5)$ & $88(0.5)$ & $98(3.1)$ \\
\hline 0.01 & $101(1.0)$ & $102(1.0)$ & $104(2.1)$ & $114(4.1)$ & $90(1.0)$ & - & - \\
\hline 0.001 & - & - & - & - & $87(2.7)$ & - & - \\
\hline
\end{tabular}

Data show host cell viability percentages (mean \pm s.e.m. of three replicates) 
Table 2 Results from the experiments with combinations of doxycycline and natural phenols on the $C$. pneumoniae infection in $\mathrm{HL}$ cells. Interaction indices, inhibition percentage of $C$. pneumoniae growth in parenthesis and host cell viability percentages in square brackets, are presented

\begin{tabular}{|c|c|c|c|c|}
\hline & \multirow[b]{2}{*}{$\mu M$} & \multicolumn{3}{|c|}{ Doxycycline } \\
\hline & & 0.07 & 0.035 & 0.018 \\
\hline \multirow[t]{5}{*}{ Quercetin } & 100 & $1.20(94)[82]$ & $2.25(68)$ [81] & $1.55(73)[78]$ \\
\hline & 50 & $2.30(51)$ [99] & 2.97 (38) [97] & $>10$ (0) [99] \\
\hline & 25 & $>10$ (14) [107] & $>10(-11)[104]$ & $>10(-29)[106]$ \\
\hline & 6.25 & $0.91(45)[105]$ & $>10(-11)[102]$ & $>10(-47)[101]$ \\
\hline & 1.25 & $>10$ (16) [107] & $>10(-21)[104]$ & $>10(-37)[106]$ \\
\hline \multirow[t]{5}{*}{ Luteolin } & 25 & $0.95(90)[81]$ & 2.09 (68) [83] & 2.95 (51) [82] \\
\hline & 12.5 & $>10(-23)[102]$ & $\begin{array}{l}>10(-80)[102] \\
>10(-111)\end{array}$ & $>10(-1)$ [102] \\
\hline & 6.25 & $>10(-1)[109]$ & [106] & $>10(-70)[106]$ \\
\hline & 1.25 & $1.57(45)$ [105] & $>10(-55)[104]$ & $>10(-45)[104]$ \\
\hline & 0.39 & $1.45(41)[100]$ & $>10(-78)[100]$ & $>10(-36)[101]$ \\
\hline \multirow[t]{5}{*}{ Rhamnetin } & 100 & $1.00(96)[94]$ & $0.85(87)$ [94] & $0.63(96)[97]$ \\
\hline & 50 & $2.17(63)$ [92] & 2.54 (36) [94] & 4.89 (18) [97] \\
\hline & 25 & $2.29(50)[91]$ & $>10(-15)[91]$ & $1.65(31)[94]$ \\
\hline & 6.25 & $2.31(26)[97]$ & $>10(-21)[102]$ & $>10(-55)[100]$ \\
\hline & 1.25 & $1.67(52)$ [102] & 2.14 (12) [102] & $>10(-47)[109]$ \\
\hline Octyl & 50 & - & - & - \\
\hline \multirow[t]{9}{*}{ gallate } & 25 & - & - & - \\
\hline & 12.5 & $>10$ (5) [90] & $>10(-21)[91]$ & >10 (14) [89] \\
\hline & 3.125 & $2.03(52)$ [103] & 2.04 (29) [105] & $4.93(7)[102]$ \\
\hline & 0.78 & 1.56 (48) [107] & $>10(-18)[113]$ & $>10(-15)[108]$ \\
\hline & & \multicolumn{2}{|c|}{ Interaction index } & Effect \\
\hline & & \multicolumn{2}{|c|}{$0-0.75$} & Synergy $^{\mathrm{a}}$ \\
\hline & & \multicolumn{2}{|c|}{$0.76-1.25$} & Additive \\
\hline & & \multicolumn{2}{|c|}{$1.26-2.00$} & Indifferent \\
\hline & & \multicolumn{2}{|c|}{$>2.00$} & Antagonism \\
\hline
\end{tabular}

a Combinations in which compound concentrations exceeded those required for maximal inhibition resulted in apparently high interaction index values.

These numeric values reflect the reaching of the upper plateau of the dose-response curves rather than antagonizing effects by the combinations.

- =host cell viability below $67 \%$.

The effect of combined treatment of infected cultures with doxycycline and the $\mathrm{Ca}^{2+}$ modulators was investigated, as this issue has earlier been addressed in persistent infection of C. pneumoniae in macrophages. ${ }^{15}$ In our model of acute C. pneumoniae infection in HL cells, the inhibitory effect of doxycycline (from 0.018 to $0.28 \mu \mathrm{M})$ was not markedly increased in the concomitant treatment of isradipine $(100,10,1 \mu \mathrm{M})$, verapamil $(100,10,1 \mu \mathrm{M})$ or thapsigargin $(1 \mu \mathrm{M}, 100$ or $10 \mathrm{~nm})$. Table 3 presents the inhibition percentages resulting from each of the combinations, together with the $I$-values calculated for the combinations. As indicated by the $I$-values, many of the combined inhibitory concentrations of doxycycline and each of the $\mathrm{Ca}^{2+}$ modulators show additive $(0.75<I \leqslant 1.25)$, however not synergistic $(I \leqslant 0.75)$, effects on inhibition of chlamydial growth. On the other hand, the combinations with subinhibitory concentrations of doxycycline and $\mathrm{Ca}^{2+}$ modulators were often indifferent $(1.25<I \leqslant 2)$ or antagonistic $($ I-values $>2)$.
The inhibition of $C$. pneumoniae by natural phenol and $\mathrm{Ca}^{2+}$ modulator combinations

The effect of combining the phenolic compounds with $\mathrm{Ca}^{2+}$ modulators were studied in similar experiments by titrating the effect of each phenolic compounds with 100,10 and $1 \mu \mathrm{M}$ isradipine or verapamil, or with $1 \mu \mathrm{M}, 100 \mathrm{~nm}$ and $10 \mathrm{~nm}$ thapsigargin. Majority of the combinations with $100 \mu \mathrm{m}$ isradipine lowered the host cell viability below $2 / 3$ of control and thus those results are not presented.

When quercetin and rhamnetin were assayed with $10 \mu \mathrm{M}$ isradipine, $I$-values of approximately 0.5 were achieved in the presence of $50 \mu \mathrm{M}$ of $100 \mu \mathrm{M}$ rhamnetin and quercetin. Thus, $10 \mu \mathrm{M}$ isradipine functioned synergistically with the high polyphenol concentrations. Furthermore, $1 \mu \mathrm{M}$ isradipine, which showed no antichlamydial effect when administered alone, was able to potentiate the antichlamydial effect of 100 and $50 \mu \mathrm{M}$ of quercetin and rhamnetin. In the case of isradipineluteolin combinations, increased numbers of chlamydial inclusions compared with untreated controls, resulting in negative inhibition values, were seen for most concentration combinations (Table 4). Thus, the $I$-values for these combinations gave values higher than 2 and were classified as antagonistic effects. Antagonism was also seen in most of the isradipine-octyl gallate combinations.

Changing the $\mathrm{Ca}^{2+}$ channel blocker from isradipine to verapamil resulted in somewhat similar results, however, with some exceptions in the case of quercetin. When the highest verapamil concentration $(100 \mu \mathrm{M})$ was combined with 0.39 and $1.56 \mu \mathrm{m}$ luteolin, $I$-values of 0.52 and 0.58 were achieved, indicating that these agents have a moderate synergistic effect in the antichlamydial assay. Verapamil $(100 \mu \mathrm{M})$ was moderately synergistic also with $1.56 \mu \mathrm{M}$ quercetin $(I-$ value 0.63$)$. Lower verapamil concentrations (10 and $1 \mu \mathrm{M})$ resulted in negative inhibition values (up to threefold number of chlamydial inclusion compared with untreated controls) independent of luteolin, quercetin (except for $100 \mu \mathrm{M}$ ) or rhamnetin concentration present in the assay media. Moreover, in the case of verapamil and octyl gallate combinations, the counts were up to fourfold compared with untreated controls, and thus heavy or moderate antagonism was indicated for all concentration combinations.

The antichlamydial effect of the three flavonoids was potentiated by thapsigargin. Thapsigargin (10 and $100 \mathrm{~nm}$ ), having no antichlamydial effect alone, resulted in $I$-values of 0.45 and 0.82 , with $6.25 \mu \mathrm{M}$ quercetin and $I$-values of 0.53 and 0.63 with $50 \mu \mathrm{m}$ quercetin, respectively. Furthermore, 10 and $100 \mathrm{~nm}$ thapsigargin were also able to strongly potentiate the antichlamydial effect of $0.39 \mu \mathrm{m}$ luteolin, providing $I$-values of 0.12 and 0.14 , respectively. Furthermore, $100 \mathrm{~nm}$ thapsigargin moderately potentiated the inhibition of $C$. pneumoniae growth when combined with 25,50 or $100 \mu \mathrm{m}$ rhamnetin, showing $I$-values of 0.3-0.7. The beneficial effects on inhibition of C. pneumoniae were less prone in the combinations of thapsigargin with octyl gallate.

\section{DISCUSSION}

Plant polyphenolic compounds, such as flavonoids, are ubiquitous in most edible plants especially tea, onion, apple, berries and grapes. They are important metabolites of plants in defense against radiation and microorganisms. ${ }^{21}$ So far, most of the antibacterial agents from nature have been derived from microbial resources, but plant metabolites deserve consideration in this respect for at least two reasons. First, plants have exceptional ability to produce cytotoxic agents and second there is an ecological rationale that antimicrobial natural products should be present or plants following microbial attack to protect the producer from pathogenic microbes in its environment. ${ }^{22}$ We have previously shown that dietary phenolic compounds are effective against the intracellular bacterium C. pneumoniae. ${ }^{17,18}$ As it 
Table 3 Results from the experiments with combinations of doxycycline and calcium modulators on the $C$. pneumoniae infection in HL cells

\begin{tabular}{|c|c|c|c|c|c|c|c|c|c|c|}
\hline & \multirow[b]{2}{*}{$\mu M$} & \multicolumn{3}{|c|}{ Isradipine } & \multicolumn{3}{|c|}{ Verapamil } & \multicolumn{3}{|c|}{ Thapsigargin } \\
\hline & & 100 & 10 & 1 & 100 & 10 & 1 & 1 & 0.1 & 0.01 \\
\hline \multirow[t]{4}{*}{ Doxycycline } & 0.28 & $2.00(94)$ [86] & $1.10(93)[96]$ & 1.01 (95) [103] & $1.33(98)$ [93] & $1.03(95)$ [97] & $1.00(85)$ [101] & $1.33(85)$ [90] & $1.03(69)$ [96] & $1.00(78)[106]$ \\
\hline & 0.07 & $1.50(96)$ [86] & 6.80 (16) [93] & $1.53(41)[100]$ & $2.2(39)$ [95] & $1.9(59)[98]$ & $>10(-13)[101]$ & 5.33 (31) [93] & 2.33 (29) [95] & $2.03(30)[105]$ \\
\hline & 0.035 & 1.25 (98) [84] & $>10(-37)[93]$ & $>10(-1)[96]$ & $>10(-7)[97]$ & $>10(-21)[103]$ & 1.99 (12) [102] & $>10$ (11) [90] & 2.94 (2) [99] & $>10(-15)[105]$ \\
\hline & 0.018 & $1.13(98)[84]$ & $>10(-10)[91]$ & $>10(-38)[100]$ & $1.76(21)$ [94] & $>10(-1)[97]$ & $>10(-36)[101]$ & 3.69 (26) [92] & 2.00 (8) [95] & $>10(-1)[107]$ \\
\hline
\end{tabular}

\begin{tabular}{cl} 
Interaction index & Effect \\
\hline $0-0.75$ & Synergy \\
$0.76-1.25$ & Additive \\
$1.26-2.00$ & Indifferent \\
$>2.00$ & Antagonism $^{\mathrm{a}}$
\end{tabular}

Interaction indices, inhibition percentage of $C$. pneumoniae growth in parenthesis, and host cell viability percentages in square brackets, are presented. ${ }^{a}$ Combinations in which compound concentrations exceeded those required for maximal inhibition resulted in apparently high interaction index values.

These numeric values reflect the reaching of the upper plateau of the dose-response curves rather than antagonizing effects by the combinations.

Table 4 Results from the experiments with combinations of calcium modulators and natural phenols on the $C$. pneumoniae infection in HL cells

\begin{tabular}{|c|c|c|c|c|c|c|c|c|c|}
\hline & \multirow[b]{2}{*}{$\mu M$} & \multicolumn{2}{|c|}{ Isradipin } & \multicolumn{3}{|c|}{ Verapamil } & \multicolumn{3}{|c|}{ Thapsigargin } \\
\hline & & 10 & 1 & 100 & 10 & 1 & 1 & 0.1 & 0.01 \\
\hline \multirow{4}{*}{ Quercetin } & 100 & 0.60 (92) [83] & $0.51(94)$ [89] & - & $0.70(90)[101]$ & 2.01 (58) [102] & $1.00(91)$ [88] & $1.05(80)$ [93] & 1. 01 (84) [92] \\
\hline & 25 & $7.00(23)[90]$ & 5.20 (13) [100] & - & $>10$ (1) [103] & $>10(-115)[104]$ & 3.33 (36) [82] & $>10(-8)[84]$ & $>10(-1)[92]$ \\
\hline & 6.25 & $>10(-85)[89]$ & $>10(-109)[95]$ & 2.68 (17) [79] & $>10(-126)[97]$ & $>10(-91)[101]$ & $1.21(52)[77]$ & $0.82(27)$ [82] & 0.45 (33) [93] \\
\hline & 1.25 & $>10(-58)[87]$ & $>10(-83)[99]$ & $0.62(46)$ [91] & $>10(-126)[96]$ & $>10(-185)[98]$ & $>10(17)[75]$ & $1.52(14)$ [79] & $>10(-9)[96]$ \\
\hline \multirow[t]{4}{*}{ Luteolin } & 12.5 & $>10(-44)[80]$ & $>10(-205)$ [89] & - & $>10(-43)[83]$ & $>10(-76)[84]$ & - & $3.33(30)[68]$ & 3.15 (35) [85] \\
\hline & 6.25 & $>10(-70)[89]$ & $>10(-242)[96]$ & - & $>10(-56)$ [92] & $>10(-91)[96]$ & $3.56(30)[77]$ & $>10(-28)[82]$ & $>10(-101)$ [92] \\
\hline & 1.25 & $>10(-152)[94]$ & $>10(-40)[97]$ & $0.58(86)[87]$ & $>10(-54)[96]$ & $>10(-13)[96]$ & $1.16(57)[73]$ & $>10(-23)[85]$ & $>10(-53)[94]$ \\
\hline & 0.39 & $>10(-163)[94]$ & $>10(-106)[95]$ & $0.52(71)$ [88] & $>10(-60)[99]$ & $>10(-10)[96]$ & $1.04(58)[70]$ & $0.14(58)$ [79] & $0.12(32)[90]$ \\
\hline \multirow{2}{*}{ Rhamnetin } & 100 & $0.60(85)$ [80] & $0.51(86)[84]$ & - & $1.07(76)[77]$ & $1.01(71)$ [83] & $1.00(100)[92]$ & 0.70 (98) [95] & $0.67(89)[84]$ \\
\hline & 1.25 & $>10(-250)[93]$ & $>10(-91)[94]$ & $>10(-124)[67]$ & $>10(-138)$ [91] & $>10(-57)[96]$ & $>10(-54)[76]$ & $>10(-126)[82]$ & $>10(-117)[88]$ \\
\hline \multirow{3}{*}{$\begin{array}{c}\text { Octyl } \\
\text { gallate }\end{array}$} & 12.5 & $0.56(85)$ [83] & $>10$ (4) [87] & 3.27 (46) [87] & $>10(-20)[84]$ & $>10(-47)[86]$ & $1.75(78)[80]$ & $1.30(75)$ [88] & $>10$ (5) [95] \\
\hline & 3.125 & $>10(0)$ [95] & $>10(-107)[100]$ & $>10$ (1) [90] & $1.88(21)[98]$ & 3.25 (12) [103] & $1.63(51)$ [81] & $>10(-17)[90]$ & $>10(-71)[96]$ \\
\hline & 0.78 & $>10(-155)[99]$ & $>10(-141)[100]$ & $>10$ (9) [90] & $>10(-289)[98]$ & $>10(-266)[102]$ & $1.16(47)[82]$ & $0.89(19)[86]$ & $>10(-54)[95]$ \\
\hline
\end{tabular}

\begin{tabular}{cl} 
Interaction index & Effect \\
\hline $0-0.75$ & Synergy \\
$0.76-1.25$ & Additive \\
$1.26-2.00$ & Indifferent \\
$>2.00$ & Antagonism
\end{tabular}

Interaction indices, inhibition percentage of $C$. pneumoniae growth in parenthesis, and host cell viability percentages in square brackets, are presented.

aCombinations in which compound concentrations exceeded those required for maximal inhibition resulted in apparently high interaction index values. These numeric values reflect the reaching of the upper plateau of the dose-response curves rather than antagonizing effects by the combinations. - =host cell viability below $67 \%$.

has been proposed that natural compounds in combination with antibiotics could be a new strategy for developing therapies for infections caused by bacterial species and that natural plant products can potentiate the activity of antibiotics in combination, ${ }^{23,24}$ we wanted to investigate whether coadministration of phenolic compounds with the antichlamydial compound doxycycline results in synergistic inhibition of acute $C$. pneumoniae infection. The combinations of natural phenols and doxycycline did not improve 
antichlamydial activity compared with the activity of compounds alone, except for $100 \mu \mathrm{M}$ rhamnetin, where additive and synergistic effects were received. However, lower concentrations of rhamnetin slightly diminished the antichlamydial effect of doxycycline. Quercetin, luteolin and octyl gallate clearly antagonized the activity of doxycycline in most of the concentration combinations. These in vitro results of the observed antagonism between doxycycline and natural phenols might give further support to the current user recommendations to avoid simultaneous intake of doxycycline with food.

The $\mathrm{Ca}^{2+}$ channel inhibitors have been shown to be of value in antimicrobial therapy by interacting with antimicrobial compounds and helping to prevent resistance. ${ }^{14,25,26}$ Azenabor et al. ${ }^{16}$ demonstrated that an L-type $\mathrm{Ca}^{2+}$ channel blocker, nifedipine, may enhance the antichlamydial effect of doxycycline in persistent infection cell model. Nifedipine treatment of macrophages infected with C. pneumoniae downregulated chlamydial hsp60 mRNA expression and upregulated major outer membrane protein expression, thus shifting the infection toward a form, which is more susceptible to antibiotic treatment. The authors concluded that the combination therapy using antibiotics and $\mathrm{Ca}^{2+}$ channel blockers should be evaluated for the treatment of chlamydial diseases. In our study, the combination of doxycycline and $\mathrm{Ca}^{2+}$ modulators against acute infection of C. pneumoniae in HL cells was at most additive and at subinhibitory concentrations of doxycycline often antagonistic.

C. pneumoniae has been shown to affect the cellular $\mathrm{Ca}^{2+}$ homeostasis in different ways. In $C$. pneumoniae-infected macrophages L-type $\mathrm{Ca}^{2+}$ channels are activated and intracellular $\mathrm{Ca}^{2+}$ concentrations are increased. ${ }^{27}$ The entry of chlamydiae has been shown to cause redistribution of cellular $\mathrm{Ca}^{2+}$ stores in HeLa cells. ${ }^{28,29}$ Local high $\mathrm{Ca}^{2+}$ concentrations and $\mathrm{Ca}^{2+}$-binding protein accumulate in the proximity of chlamydial aggregates and they are pivotal for the proper formation of chlamydial inclusions. In a recent microarray study by Alvesalo et al., ${ }^{30}$ expression levels of genes associated with regulation of $\mathrm{Ca}^{2+}$ homeostasis were significantly changed by $C$. pneumoniae infection in $\mathrm{HL}$ cells. The results presented in the current work indicate that $\mathrm{Ca}^{2+}$ modulators alone have only a minor effect on acute $C$. pneumoniae infection in HL cells, or in the case of verapamil, may even increase the number of chlamydial inclusions.

When studying phenolic compounds and $\mathrm{Ca}^{2+}$ modulators, more antagonistic combinations were found than synergic or additive combinations. However, $10 \mu \mathrm{M}$ isradipine was synergistic with high quercetin and rhamnetin concentrations $(100 \mu \mathrm{M}$ and $50 \mu \mathrm{M})$, whereas in the presence of low quercetin/rhamnetin concentrations, the combinations increased the inclusion counts. Verapamil $(100 \mu \mathrm{M})$ was synergistic with low quercetin and luteolin concentrations, but unfortunately, compromised cell viability limits the use of higher polyphenol concentrations. Although thapsigargin had no antichlamydial effect on its own, it was able to potentiate the growth inhibitory effect of quercetin, luteolin and rhamnetin on C. pneumoniae.

To summarize our results, monotherapy with $\mathrm{Ca}^{2+}$ modulators was not inhibitory on chlamydial growth. Verapamil (10 and $1 \mu \mathrm{M})$ increased the number of $C$. pneumoniae inclusions in the acute infection model, and this effect was generally not reversed by inhibitory concentrations of the phenolic compounds. However, verapamil did not abolish the antichlamydial effect of doxycycline. High concentrations of quercetin and rhamnetin combined with isradipine and thapsigargin showed clear positive effects on the inhibition of $C$. pneumoniae, whereas other combinations of $\mathrm{Ca}^{2+}$ modulators and phenolic compounds only occasionally resulted as improved inhibition.
To conclude, high degree of dose dependence was associated with the observed effects in all cases. The coadminstration of $\mathrm{Ca}^{2+}$ modulators with phenolic compounds resulted in synergistic inhibition of C. pneumoniae growth, whereas combination of these with doxycycline resulted in additive effects at best. Coadministration of phenolic compounds with doxycycline provided no marked beneficial effects. The present study suggest on the potential usefulness of phenolic compounds and $\mathrm{Ca}^{2+}$ modulators in combination for combating infections caused by this pathogen.

\section{MATERIALS AND METHODS}

\section{Cell lines}

HL cell line was used as host cells for the C. pneumoniae infection. The cells were grown as described in Salin et al. ${ }^{31}$ Briefly, the culture medium consisted of RPMI 1640 supplemented with $7.5 \%$ fetal bovine serum and $2 \mathrm{~mm}$ L-glutamine, all purchased from BioWhittaker, Lonza (Basel, Switzerland) and with $20 \mu \mathrm{g}$ gentamycin (Fluka, Buchs, Switzerland) per ml. Cells were cultured to confluence in culture flasks (Greiner, Bio-One, Frickenhausen, Germany) in standard cell culture conditions $\left(37^{\circ} \mathrm{C}, 5 \% \mathrm{CO}_{2}\right.$ and $95 \%$ humidity). The Chlamydia strain used was a sequenced ATCC strain CWL-029 (VR-1310) and was grown and purified as described in Alvesalo et al. ${ }^{17}$

\section{Compounds}

The natural compounds, luteolin and rhamnetin were purchased from Extrasynthese (Genay, France), quercetin from Carl Roth GmbH (Karlsruhe, Germany) and octyl gallate from Fluka. Doxycycline was from ICN Biomedicals (Aurora, OH, USA) and verapamil and isradipine from Sigma-Aldrich (St Louis, MO, USA). Thapsigargin was a generous gift from Professor Kid Törnquist at Abo Akademi University, Turku, Finland. All stock solutions were made in DMSO (Mallinckrodt Baker, Phillipsburg, NJ, USA) at a concentration of $100 \mathrm{~mm}$, except for doxycycline $(0.56 \mathrm{~mm})$ and thapsigargin $(1 \mathrm{~mm})$.

\section{Antichlamydial assays}

The antichlamydial assays were performed by an immunofluorescence method on a 24-well plate, quantifying the number of chlamydial inclusions in infected HL cells using a Chlamydia-specific anti-LPS antibody, as described earlier in Salin et al. ${ }^{31}$ and Keurulainen et al. ${ }^{32}$

The antichlamydial effect of compound combinations was assayed by using a checkerboard method, with a single replicate. ${ }^{33}$ Combinations were chosen, based on the activity results from dose-response experiments for single agents, and on each of the combination plates, the compounds were also present alone.

\section{Cell viability assays}

The effect of different concentrations of single compounds $(n=3)$ and the studied combinations in different concentrations $(n=1$ for each concentration combination) on the host cell viability was determined using the luminescent ATP level determination method as described in Salin et al. ${ }^{31}$ All chemical treatments were well tolerated by the host cells $(67 \%$ viability or higher of DMSO-treated control), unless otherwise stated in the text or tables.

\section{Data analysis}

All antichlamydial results represent mean and s.e.m. values from the number of replicates indicated for each experiment. The sigmoidal dose response curves and the $\mathrm{IC}_{50}$ values were determined by fitting the data from experiments with concentration series into nonlinear regression models using the GraphPad Prism software version 5.0. (GraphPad Software, Inc., La Jolla, CA, USA) For the analysis of concomitant administration of the chemical agents, Loewe model for additivity was applied by calculating the $I$-values for each combined dose. The equation for calculating $I$ was as follows:

$$
\mathrm{I}=\mathrm{D}_{1} / \mathrm{D}_{\mathrm{X} 1}+\mathrm{D}_{2} / \mathrm{D}_{\mathrm{X} 2}
$$

where $D_{1}$ and $D_{2}$ represent the concentrations of compounds in the combination, and $D_{\mathrm{X} 1}$ and $D_{\mathrm{X} 2}$ represent the concentrations of the drugs alone giving similar response. 


\section{ACKNOWLEDGEMENTS}

We acknowledge the Sigrid Juselius Foundation, Tor, Joe och Pentti Borgs Minnesfond Foundation and the Finnish Cultural Foundation (Elli Turunen) for financial support.

1 Kuo, C. C., Jackson, L. A., Campbell, L. A. \& Grayston, J. T. Chlamydia pneumoniae. Clin. Microbiol. Rev. 8, 451-461 (1995).

2 Rassu, M. et al. Demonstration of Chlamydia pneumoniae in atherosclerotic arteries from various vascular regions. Atheroscerosis 158, 73-79 (2001).

3 Cao, F., Castrillo, A., Tontonoz Re, F. \& Byrne, G. I. Chlamydia pneumoniae-induced macrophage foam cell formation is mediated by Toll-like receptor 2. Infect. Immun. 75, 753-759 (2007).

4 Blessing, E., Campbell, L. A., Rosenfeld, M. E., Chough, N. \& Kuo, C. C. Chlamydia pneumoniae infection accelerates hyperlipidemia induced atherosclerotic lesion development in C57BL/6J mice. Atherosclerosis 158, 13-17 (2001).

5 Volanen, I. et al. Increased aortic intima-media thickness in 11-year-old healthy children with persistent Chlamydia pneumoniae seropositivity. Arterioscler. Thromb. Vasc. Biol. 26, 649-655 (2006).

6 Kohanski, M., Dwyer, D. \& Collins, J. J. How antibiotics kill bacteria: from targets to networks. Nat. Rev. Microbiol. 8, 423-435 (2010).

7 Carter, J. D. et al. Combination antibiotics as a treatment for chronic Chlamydiainduced reactive arthritis. Arthritis. Rheum. 62, 1298-1307 (2010).

8 Kutlin, A., Roblein, P. M. \& Hammerschlag, M. R. Effect of prolonged treatment with azithromycin, clarithromycin or levofloxacin on Chlamydia pneumoniae in a continuousinfection model.. Antimicrob. Agents Chemother. 46, 409-412 (2002).

9 Grayston, J. T. Infections caused by Chlamydia pneumoniae strain TWAR. Clin. Infect. Dis. 15, 757-763 (1992).

10 Gieffers, G., Rupp, J., Gebert, A., Solbach, W. \& Klinger, M. First-choice antibiotics at subinhibitory concentrations induce persistence of Chlamydia pneumoniae.. Antimicrob. Agents Chemother. 48, 1402-1405 (2004).

11 Bellman-Weiler, R. et al. Divergent modulation of Chlamydia pneumoniae infection cycle in human monocytic and endothelial cells by iron, tryptophan availability and interferon gamma. Immunobiology 215, 842-848 (2010).

12 Gieffers, G. et al. Chlamydia pneumoniae infection in circulating human monocytes is refractory to antibiotic treatment. Circulation. 103, 351-356 (2001).

13 Mazumdar, K., Kumar, K. A. \& Dutta, N. K. Potential role of the cardiovascular nonantibiotic (helper compound) amlodipine in the treatment of microbial infections: scope and hope for the future. Int. J. Antimicrob. Agents 36, 295-302 (2010).

14 Shainkin-Kestenbaum, R., Winikoff, Y., Kol, R., Chaimovizt, C. \& Sarov, I. Inhibition of growth of Chlamydia trachomatis by the calcium antagonist verapamil. J. Gen. Microbiol. 135, 1619-1623 (1989).

15 Azenabor, A. A. \& Chaudhry, A. U. Macrophage L-type $\mathrm{Ca}^{2+}$ channel antagonists alter Chlamydia pneumoniae MOMP and HSP-60 mRNA gene expression, and improve antibiotic susceptibility. Immunobiology 207, 237-245 (2003).
16 Azenabor, A. A. Treatment of chronic bacterial infections Pat. Pub. No: WO 2005/ 007082. International Application No.: PCT/US2004/016780.

17 Alvesalo, J., Vuorela, H., Tammela, P., Leinonen, M., Saikku, P. \& Vuorela, P. Inhibitory effect of dietary phenolic compounds on Chlamydia pneumoniae in cell cultures. Biochem. Pharmacol. 71, 735-741 (2006).

18 Törmäkangas, L. et al. In vivo treatment of acute Chlamydia pneumoniae infection with the flavonoids quercetin and luteolin and an alkyl gallate, octyl gallate, in a mouse model. Biochem. Pharmacol. 70, 1222-1230 (2005).

19 Scholz, E. P., Zitron, E., Katus, H. A. \& Carle, C. A. Cardiovascular ion channels as a molecular target of flavonoids. Cardiovasc. Ther. 28, 46-52 (2010)

20 Summanen, J. et al. Effects of simple aromatic compounds and flavonoids on $\mathrm{Ca}^{2+}$ fluxes in rat pituitary $\mathrm{GH}_{4} \mathrm{C}_{1}$ cells. Eur. J. Pharmacol. 414, 125-133 (2001).

21 Cushnie, T. P. T. \& Lamb, A. G. Antimicrobial activity of flavonoids. Int. J. Antimicrob. Agents 26, 343-356 (2005).

22 Gibbons, S. Plants as a source of bacterial resistance modulators and anti-infective agents. Phytochem. Rev. 4, 63-78 (2005).

23 Lewis, K. \& Ausubel, F. M. Prospects for plant-derived antibacterial. Nat. Biotechnol. 24, 1504-1507 (2006).

24 Jayaraman, P., Sakharkar, M. K., Lim, C. S., Tang, T. H. \& Sakharkar, K. R. Activity and interactions of antibiotic and phytochemical combinations against Pseudomonas aeruginosa in vitro. Int. J. Biol. Sci. 6, 556-568 (2010).

25 Liebermann, L. A. \& Higgins, D. E. Inhibition of Listeria monocytogenes infection by neurological drugs. Int. J. Antimicrob. Agents 35, 292-296 (2010).

26 Gunics, G., Motohasi, N., Amaral, L., Farkas, S. \& Molnár, J. Interaction between antibiotics and non-conventional antibiotics on bacteria. Int. J. Antimicrob. Agents 14, 239-242 (2000).

27 Azenabor, A. \& Chaudry, A. Effective macrophage redox defence against Chlamydia pneumoniae depends on L-type $\mathrm{Ca}^{2+}$ channel activation. Med. Microbiol. Immunol. 192, 99-106 (2003).

28 Majeed, M., Krause, K.- H., Clark, R., Kihlström, E. \& Stendahl, O. Localization of intracellular $\mathrm{Ca}^{2+}$ stores in HeLa cells during infection with Chlamydia trachomatis. J. Cell Sci. 112, 35-44 (1999).

29 Majeed, M., Ernst, J., Magnusson, K.- E., Kihlström, E. \& Stendahl, O. Selective translocation of annexins during intracellular redistribution of Chlamydia trachomatis in HeLa and McCoy cells. Infect. Immun. 62, 126-134 (1994).

30 Alvesalo, J. et al. Microarray analysis of a Chlamydia pneumoniae-infected human epithelial cell line by use of gene ontology hierarchy. J. Infect. Dis. 197, 156-162 (2008).

31 Salin, 0. et al. Inhibitory effect of the natural product betulin and its derivatives against the intracellular bacterium Chlamydia pneumoniae. Biochem. Pharmacol. 80, 1141-1151 (2010).

32 Keurulainen, L. et al. Design and synthesis of 2-arylbenzimidazoles and evaluation of their inhibitory effect against Chlamydia pneumoniae. J. Med. Chem. 53, 7664-7674 (2010).

33 How, S. J., Hobson, D., Hart, A. C. \& Webster, R. E. An in vitro investigation of synergy and antagonism between antimicrobials against Chlamydia trachomatis. J. Antimicrob. Chemother. 15, 533-538 (1985). 